\title{
Diagnóstico de las Actividades Pesqueras en la Región Tacna, Periodo 1998-2007
}

RESPONSABLE: Blgo. Pesq. Nelver Coronel Flores

\begin{abstract}
RESUMEN En el presente artículo se realiza el análisis de las diversas actividades pesqueras desarrolladas en el ámbito marino y continental de la región Tacna. La pesquería en esta región es de carácter netamente artesanal, no existe pesca industrial. El producto de la pesqueria marítima (peces, moluscos, crustáceos y equinodermos) está destinado mayormente para el consumo humano directo. Los únicos recursos hidrobiológicos utilizados en el procesamiento o transformación, además de las ovas de erizo de mar Loxechinus albus y de pez volador, es la anchoveta Engraulis ringens, exportada con el nombre de "anchoa". En el ámbito continental la actividad pesquera se circunscribe a la extracción de trucha arco iris Oncorhynchus mykiss en los ríos y lagunas altoandinas $y$ del recurso camarón de rio Cryphiops caementarius en la cuenca baja del rio Sama.
\end{abstract}

\begin{abstract}
In the present report of the research working called "Diagnostics of fishing activities in the region Tacna, period 1998-2007", the analysis of the various fishing activities developed at marine and continental environment of region Tacna is made. The fishery in this region is purely craft, there is no industrial fishing. The product of the maritime fishery (fish, molluscs, crustaceans and echinoderms) are intended mainly for the direct human consumption. The unique hydrobiological resources used in the processing or transformation are sea urchin ovas Loxechinus albus, flying fish ovas and anchovy Engraulis ringens, exported named "anchoa". At the continental level the fishing is confined to the extraction of trout rainbow Oncorhynchus mykiss into rivers and lakes located high altitude and the river shrimp resource Cryphiops caementarius in the low the Sama river basin.
\end{abstract}

INTRODUCCIÓN En el ámbito de la región Tacna la actividad pesquera es netamente de carácter artesanal. Esta es una actividad con altos niveles de incidencia en la economía, no solamente local (regiones costeñas), sino también nacional, por su contribución a los índices de empleo y el aporte a la seguridad alimentaria de la población. Sin embargo, esta actividad atraviesa por una etapa de crisis, agravada por la sobreexplotación y la progresiva disminución de los recursos hidrobiológicos. Esta situación pone en riesgo la sobrevivencia de miles de familias cuyos ingresos dependen directa e indirectamente de los niveles de producción de la pesca artesanal.

Conocer las características de las diversas actividades pesqueras, en sus diferentes fases (extracción, cultivo, procesamiento y comercialización) es de vital importancia dentro del concepto de sostenibilidad de los recursos explotables. En la región Tacna no existen a la fecha estudios actualizados sobre las interrelaciones entre los distintos agentes involucrados en dichas actividades pesqueras, de ahí que es muy importante y se justifica la realización del presente estudio, el cual permitirá al final contar con los elementos técnicocientíficos necesarios para proponer una explotación racional y sostenida de los recursos hidrobiológicos de la región Tacna.

Para la elaboración del presente documento se ha recurrido a la recopilación de diversas fuentes estadísticas, tales como los anuarios estadísticos publicados por la Dirección Regional de la Producción de Tacna, el Instituto Nacional de Estadística - Tacna, Dirección Regional de Comercio Exterior y Turismo de Tacna, Superintendencia Nacional de Administración Tributaria (SUNAT) y Cámara de Comercio de Tacna.

\section{MATERIALY MÉTODOS}

En la ejecución del presente trabajo de Investigación se utilizaron los siguientes materiales y equipos: formularios especialmente diseñados para la recopilación de información de campo, computadora personal Core 2 Duo, geoposicionador satelital (GPS), cámara fotográfica digital y cámara filmadora.

La metodología fue la siguiente:

Trabajos de campo.

Los trabajos de campo consistieron en el acopio de toda la información relacionada con la actividad pesquera disponible en las diferentes entidades (Dirección Regional de la Producción de Tacna, Instituto Nacional de Estadística de Tacna, Gobierno Regional de Tacna, etc.) y agentes involucrados con el quehacer pesquero (asociaciones de pescadores artesanales de Tacna, 
asociaciones de comerciantes de productos hidrobiológicos de Tacna y empresas industriales pesqueras de Tacna).

\section{Trabajos degabinete.}

En el gabinete se realizaron los análisis, interpretación, discusión y procesamiento de toda la información obtenida en el campo, haciendo uso de un computador Core 2 Duo, empleando softwares de uso común para este tipo de trabajos, tales como Word, Excel y softwares estadísticos (SPPS y SYSTAT).

\section{RESULTADOSYDISCUSIÓN}

\section{Desembarque de Recursos Hidrobiológicos en Tacna}

A los recursos hidrobiológicos desembarcados en Tacna, para fines estadísticos, la Dirección Regional de la Producción de Tacna los clasifica según su destino o rubro en : fresco, congelado, enlatado y curado. Las especies empleadas en cada rubro son las siguientes:

Al estado fresco: pescado: anchoveta, berrugate, bonito, caballa, cabinza, cabrilla, coco, cojinova, corvina, jurel, lenguado, lisa, lorna, machete, perico, pejerrey, raya, sardina, sargo, tiburón azul, tiburón diamante, tollo y trucha; mariscos: almeja, calamar/pota, camarón de río, caracol, choro, jaiva, lapa,macha, pulpo y tolina.

Al estado congelado: pescado: machete, perico, pejerrey y sardina; mariscos: caracol, erizo de mar, lapa, macha, pota, pulpo, tolina y jaiva.

Enlatado: pescado: machete; mariscos: caracol, lapa, macha y tolina.

Curado: pescado: anchoveta; mariscos: macha y pepino demar.

Del total de especies hidrobiológicas desembarcadas y comercializadas en Tacna, la mayoría son peces (23 especies), de los cuales la mayor parte son destinados al consumo humano directo al estado fresco; únicamente la anchoveta es el pez que viene siendo procesado en forma de curado. Los denominados "mariscos" ocupan el segundo lugar en cuanto a su diversidad específica.

Los montos anuales del desembarque de recursos hidrobiológicos para el consumo humano directo (CHD) en Tacna, durante los últimos diez (10) años (1998-2007) se especifica en el Cuadro $\mathrm{N}^{\circ} 01$.
Cuadro $\mathrm{N}^{\circ} 01$ Desembarque anual de recursos hidrobiológics para consumo humano directo en Tacna (TMB), según rubros.-De 1998 a 2007.

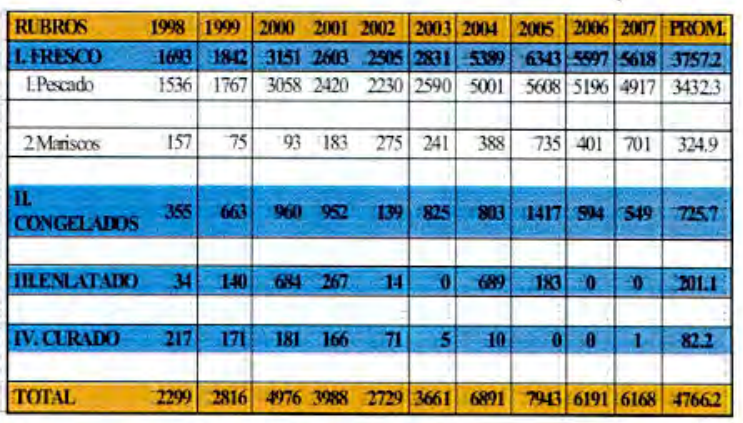

Fuente: Dirección Regional de la Producción de Tacna.

Los desembarques de los recursos hidrobiológicos para CHD en Tacna, en forma general, se han incrementado paulatinamente a través de los años. Así, en el año 1998 se desembarcó un total de 2299 TMB, fue incrementándose hasta que en el año 2000 Hegó a 4976 TMB, luego fue desciendo hasta el año 2002, con 2729 TMB. En los últimos cuatro años, los montos de desembarque se han incrementado hasta llegar a su punto más alto en el año 2005 con la cifra record de 7943 TMB. En los años 2006 y 2007, el monto total de desembarque fue de 6191 y 6168 TMB, respectivamente.

Los mayores montos desembarcados corresponden a los recursos hidrobiológicos destinados al consumo en fresco, con valores que van desde 1693 TMB, ocurrido en el año 1998 hasta el máximo de 6343 TMB, que se presentó en al año 2005 (Cuadro $\mathrm{N}^{\circ} 01$ ). Le siguen los desembarques destinados al proceso de congelado, con montos que oscilaron desde las 139 TMB (año 2002) hasta las 1417 TMB (año 2005). Los montos destinados al proceso de fabricación de conservas (enlatado) también presentaron oscilaciones acentuadas a través del período analizado; los máximos valores se presentaron en los años 2000 y 2004 , con 684 y 689 TMB, respectivamente; en el año 2005 el desembarque para enlatado descendió bruscamente hasta un valor de 183 TMB; en los años 2006 y 2007 no hubo desembarques para enlatado. Los montos destinados al proceso de curado son mínimos en comparación de los otros rubros, con valores desde 1.0 TMB (año 2007) hasta un máximo de 217 TMB (año 1998); en los años 2005 y 2006 no hubo desembarque para curado.

En la década de estudio (1998 - 2007), la composición de los desembarques de los recursos hidrobiológicos por grupos taxonómicos fue: pescado, que representa la mayor cantidad ( $76,48 \%$ del total del desembarque), los moluscos $(22,92 \%)$, crustáceos $(0,34)$ y equinodermos $(0,26 \%)$ (Gráf. $\left.N^{\circ} 1\right)$. 


\section{Extracción de Recursos Hidrobiológicos Continentales en} Tacna

Los únicos recursos hidrobiológicos que son explotados en los recursos hídricos continentales de la región Tacna son el camarón de río Cryphiops caementarius y la trucha arco iris Oncorhynchus mykiss. Los montos de extracción y, por consiguiente, de desembarque se detallan en el Cuadro $\mathrm{N}^{\circ} 02$.

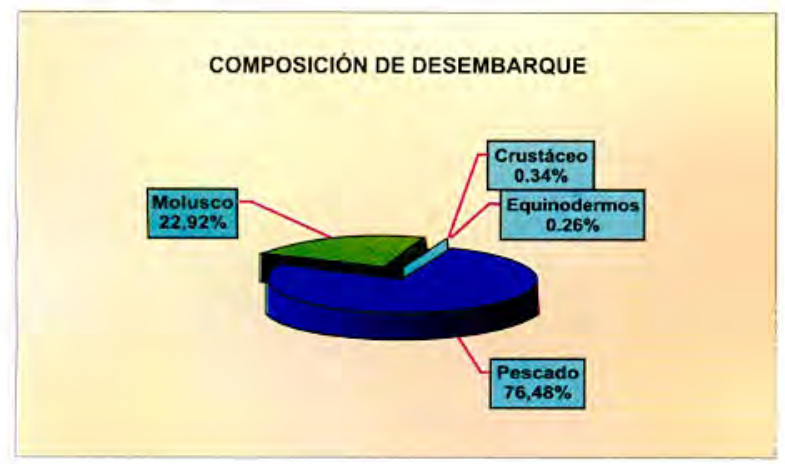

Gráfica $\mathrm{N}^{0} 1$ Composición porcentual de los desembarques de recursos hidrobiológicos para consumo humano directo en Tacna

Cuadro $\mathrm{N}^{\circ} 02$ Desembarque de recursos hidrobiológicos (TM) de origen continental en la región Tacna-De 1997 al 2006.

\begin{tabular}{|c|c|c|}
\hline AÑo & CAMARÓN DE RÍO & TRUCHA ARCO IRIS \\
\hline $\mathbf{1 9 9 7}$ & 3,1 & 56,2 \\
$\mathbf{1 9 9 8}$ & 2,3 & 59,0 \\
$\mathbf{1 9 9 9}$ & 1,5 & 69,0 \\
$\mathbf{2 0 0 0}$ & 3,8 & 65,0 \\
$\mathbf{2 0 0 1}$ & 3,0 & 48,5 \\
$\mathbf{2 0 0 2}$ & 1,6 & 42,8 \\
$\mathbf{2 0 0 3}$ & 3,3 & 54,5 \\
$\mathbf{2 0 0 4}$ & 5,9 & 35,3 \\
$\mathbf{2 0 0 5}$ & 5,6 & 34,1 \\
$\mathbf{2 0 0 6}$ & 5,4 & 22,3 \\
\hline PROM. & $\mathbf{3 , 6}$ & $\mathbf{4 8 , 7}$ \\
\hline
\end{tabular}

Fuente: Dirección Regional de la Producción de Tacna.

Los montos de extracción o desembarque de camarón y trucha son fluctuantes a través de los años. Los desembarques anuales de camarón, durante el periodo evaluado, presentó un valor mínimo de 1,5 TM, que se presentó en el año 1999 y un máximo de 5,9 TM que ocurrió en el año 2004. Es necesario mencionar que las capturas de este crustáceo se han venido incrementando en estos tres últimos años.

Por su parte, el desembarque anual de trucha, para el período evaluado, es mucho mayor que el del camarón, pero, a diferencia de lo que ocurre con el camarón, ha ido decreciendo desde el máximo valor de 69 TM, que se presentó en el año 1999 hasta el mínimo de 22,3 TM desembarcadas en el año 2006.

\section{Consumo de Recursos Hidrobiológicos en Tacna}

El consumo de recursos hidrobiológicos en Tacna durante el período de estudio (1998-2007), a diferencia del desembarque, no presentó variaciones anuales acentuadas. Los montos oscilaron desde $1052 \mathrm{TMB}$, que ocurrió el año 1998 hasta 1701.0 TMB, que se presentó en el año 2006.

Por otro lado, la mayoría de recursos hidrobiológicos que se consumen en Tacna son al estado fresco. Los rubros enlatado (conservas) y curado son poco significativos y el grupo de congelados simplemente no se consumen en Tacna.

Las especies de pescado que tuvieron mayor consumo en el período analizado, en orden de importancia, son: "caballa", con un promedio anual de 205 TM, "jurel" (198 TM), "lorna" (153 TM), "pejerrey" (113 TM), "perico" (77 TM), "cabinza" (73 TM), "lisa" (33 TM) y "tiburón diamante" (30 TM). El resto de especies no superan las $30 \mathrm{TM}$ anuales de consumo.

Dentro de los denominados "mariscos", el "calamar"o "pota" es el que ha tenido el mayor consumo en el período analizado (46 TM de promedio anual), le siguen el "choro" (45 TM), la "jaiva" (34 TM), la "lapa" (11 TM), el "caracol" (9 TM), el camarón de río (8 TM), el "pulpo" (7 TM), la "almeja" (7 TM) y el "chanque" o "loco" (1 TM). Cabe indicar que el consumo de "chanque" en Tacna es relativamente poco, ya que la mayor parte de este molusco es destinado al procesado (congelado y conservas) y colocado en el mercado exterior. El bivalvo "macha", que fue un recurso muy importante hasta hace aproximadamente 10 años, ha desaparecido del litoral de Tacna. Hay reportes estadísticos de su consumo solamente hasta el año 1998.

Además se puede concluir que, el consumo de pescado al estado fresco en Tacna representa aproximadamente el $80 \%$ del consumo total de recursos y productos hidrobiológicos, frente al $14 \%$ que está dado por los mariscos. Los recursos hidrobiológicos procesados en forma de conservas, curado y congelado, representan en conjunto únicamente el $6 \%$ del consumo total.

\section{Procesamiento de Recursos Hidrobiológicos en Tacna}

El procesamiento de recursos hidrobiológicos en Tacna es efectuado por las empresas industriales pesqueras ubicadas en el Parque Industrial, comprendiendo los rubros de enlatado (conservas), congelados (básicamente tipo IQF), refrigerados y curado. Los dos primeros rubros son los más significativos, especialmente los congelados.

Los montos de los recursos hidrobiológicos procesados, durante el período evaluado, ha sido fluctuante, desde un mínimo de 1645 TMB (1999) hasta un máximo de 4575 TMB (2005). En el año 2007 fue de $4346 \mathrm{TMB}$. 


\section{Comercialización Externa (Exportación) de Productos Pesqueros de Tacna}

Los productos pesqueros elaborados en Tacna y que son comercializados en el mercado exterior (exportación), en orden de importancia son el "chanque" (hasta el año 2005), seguido de la "lapa", "caracol", "pota", "pejerrey", "perico" "anchoveta" y gónadas de "erizo", "jaiva" y "pez volador", en forma de enlatados (conservas), congelado (tipo IQF), refrigerado y curado.

En el año 2001 el monto exportado fue de 365,2 TMB, al año siguiente subió a 769,1 TMB, bajó ligeramente en el año 2003 a 681,6 TMB, volviendo a subir en el año 2004 a 1702,5 TMB. En el año 2005, la exportación subió notablemente hasta la cifra record de 2860,4 TMB.

Los productos congelados son los que ostentan los mayores volúmenes de exportación. En el año 2001 se exportó un total de 176,6 TMB, en los años siguientes se fue incrementando hasta llegar en el año 2005 a la cifra record nunca antes reportada de 1906,6 TMB.

El volumen exportado de conservas igualmente se ha ido incrementando con el transcurso de los años. En el año 2001 se exportó un total de 50.6 TMB y en el 2005, un total de 897,2 TMB.

En cuanto a los montos de refrigerado y curado, estos son mínimos, con valores que van desde 17 TMB hasta un máximo de $113 \mathrm{TMB}$; incluso, en el año 2004 no se exportaron productos hidrobiológicos bajo el rubro de curado.

\section{Comercialización Interna de Productos Pesqueros de Tacna}

Los productos pesqueros procesados bajo los rubros de conserva y congelado en Tacna y destinados al mercado interno (nacional) están representados mayormente por otras especies diferentes al "chanque", tales como sardina, caballa, jurel, pejerrey, pulpo, caracol y pota. Los montos de estos productos son relativamente bajos en comparación con los destinados al mercado exterior.

Los montos de la comercialización interna de los productos pesqueros de Tacna, a diferencia de los de la comercialización externa, han ido disminuyendo a través de los años; así se tiene que, en el año 2001 se comercializó un total de 1254,3 TMB, al año siguiente bajó a 881,9 TMB, siguió disminuyendo en los años posteriores hasta su valor más bajo en el año 2004 , con únicamente 10,4 TMB. El último año registrado (2005) se recuperó, alcanzando un valor de 45,7 TMB.

\section{CONCLUSIONES}

1. La actividad pesquera extractiva en la región Tacna es básicamente artesanal y orientada al consumo humano directo, a través de los rubros fresco/refrigerado, congelado, enlatado y curado.
2. Las especies hidrobiológicas de mayor consumo al estado fresco son: pescado: jurel, caballa, perico, lorna, sargo, diamante, azul y raya; mariscos: lapa, choro, jaiva, almeja y tolina.

3. Los desembarques de productos hidrobiológicos en Tacna en la última década (1998-2007) han tenido en forma general un ascenso, pasando de las 2299 TM que se desembarcaron en el año 1998 hasta la cifra récord de $7946 \mathrm{TM}$, en el año 2005.

4. La composición de los desembarques de los recursos hidrobiológicos por grupos taxonómicos fue: pescado ( $76,48 \%$ del total del desembarque), los moluscos $(22,92 \%)$, crustáceos $(0,34)$ y equinodermos $(0,26 \%)$

5. Los desembarques anuales de camarón, durante el período evaluado, presentaron un valor mínimo de 1,5 TM, que se presentó en el año 1999 y un máximo de 5,9 TM que ocurrió en el año 2004.

6. El desembarque anual de trucha, para el período evaluado, es mucho mayor que el del camarón, pero, a diferencia de lo que ocurre con el camarón, ha ido decreciendo desde el máximo valor de 69 TM, que se presentó en el año 1999 hasta el mínimo de 22,3 TM desembarcadas en el año 2006.

7. En la ciudad de Tacna, mayormente se consume pescado al estado fresco, poca cantidad de conservas (enlatado) y mucho menos de seco-salado (curado). El consumo de productos hidrobiológicos congelados es insignificante.

8. Los productos congelados son los que ostentan los mayores volúmenes de exportación. En el año 2001 se exportó un total de 176,6 TMB, en los años siguientes se fue incrementando hasta llegar en el año 2005 a la cifra récord nunca antes reportada de 1906,6 TMB.

9. Los montos de la comercialización interna de los productos pesqueros de Tacna, a diferencia de los de la comercialización externa, han ido disminuyendo a través de los años. En el año 2001 se comercializó un total de 1254,3 TMB, al año siguiente bajó a 881,9 TMB, siguió disminuyendo en los años posteriores hasta su valor más bajo en el año 2004, con únicamente 10,4 TMB. 